\title{
THE EFFECT OF LLLT ON THE HARD AND SOFT TISSUES OF THE JAW BONES
}

\author{
Petko Petrovi ${ }^{1}$, Tsvetan Tonchev ${ }^{2}$ \\ ${ }^{1}$ Department of Maxillofacial Surgery, Faculty of Dental Medicine, \\ Medical University - Plovdiv \\ ${ }^{2}$ Department of Oral and Maxillofacial Surgery and Special Imaging Diagnostics, \\ Faculty of Dental Medicine, Medical University of Varna
}

\begin{abstract}
INTRODUCTION: Dental implantology is developing rapidly. Surgical procedures related to this type of therapy require a long recovery period. The application of regeneration-stimulating procedures would reduce this period.

AIM: The aim of this study is to present the results of modern research on the stimulating effect of Low-Level Laser Therapy (LLLT) on bone recovery and on the treatment of soft tissues.

MATERIAL: An extensive analysis is made of the results in 28 literature sources on the interaction between LLLT and implant osseointegration and the quality of the surrounding soft tissues.

CONCLUSION: Laser irradiation has a broad impact spectrum on tissues and a single overexposure or lower level exposure may change significantly clinical results. Adopting the appropriate method of stimulation is of major importance.
\end{abstract}

Keywords: low-level laser therapy (LLLT), titanium implants, osseointegration, hard tissues, soft tissues

\section{INTRODUCTION}

In their lifetime, for a variety of reasons, people lose some or all of their teeth, which leads to serious physiological and psychological problems. The various defects and deformities thus obtained disturb the individual's vital functions. More and more frequently, modern dental medicine is using dental implants and supports for prosthetic restorations (13).

Address for correspondence:

Petko Petrov

Department of Maxillofacial Surgery,

Faculty of Dental Medicine,

Medical University - Plovdiv

66 Peshtersko Shose Str.

4000 Plovdiv

e-mail:petkopetrov@abv.bg

Received: December 9, 2015

Accepted: December 15, 2015
Surgical procedures related to this type of therapy require a long recovery period. The application of regeneration-stimulating procedures would reduce this period.

\section{AIM}

The aim of this study is to present the results of modern research on the stimulating effect of LowLevel Laser Therapy (LLLT) on bone recovery and on the treatment of soft tissues.

\section{MATERIAL}

LLLT has been in use for over 30 years and no existing adverse effects have been reported. During the last decade, an increase has been registered in the use of LLLT in dental practices. Experimental studies conducted on rats have shown a significant rise of the alkaline phosphatase level 6 days after the irradiation of standard bone defects, which is an indicator of increased osteoblastic activity. 
Another research has proved that low-level laser irradiation improves vascularization and raises the number of trabeculae in cases of fractured tibia in laboratory mice (3). During an in vitro experiment conducted with rat osteoblasts, three low-level laser irradiation sessions increased significantly the number of osteoblasts after an interval of 8 days (1). The LLLT-associated effects include blood flow stimulation, osteoblast and osteosynthesis restoration and activation, reduction of osteoclastic activity and antiinflammatory effect (20).

Biostimulation conducted through LLLT is a particular area of disagreement. Many studies have shown a positive effect of the application of low-level laser irradiation in bone reconstruction, both in vivo (6), and in vitro (26). However, there are other studies which do not discover any effect of LLLT on soft and hard tissue healing. Despite this, the number of studies registering a positive effect is significantly larger $(15,16)$.

There are studies which present evidence of the effect of LLLT on soft tissues (11,12,14,17-19). Just like with normal epithelium, the healthy mucous membrane has a significant role in protecting the peri-implant sulcus from bacterial invasion. The peri-implant soft tissues however are more unstable than the natural periodontal soft tissues. The absence of attachment around the implants may cause peri-implantitis and therefore lead to unsuccessful outcome of the therapy. Mucous membrane regeneration and the creation of intact functional barrier in transmucosal penetration of the implant support is one of the advantages of the low-level laser therapy. In a recently conducted research, Khadra $\mathrm{M}$ et al. (9) study the effect of Gallium-Aluminium-Arsenide (Ga-Al-As) diode laser on the proliferation and attachment of human gingival fibroblasts (HGF), cultivated on titanium implant material. The main result is that the percentage of attachment of the fibroblasts exposed to laser irradiation is significantly higher than the cells which have not been exposed to laser irradiation. What is more, the irradiated cells demonstrate significantly higher level of proliferation. The study also reveals that raising the dose from 1.5 to $3 \mathrm{~J} \mathrm{~cm}^{2}$ does not cause further rise in the initial HGF reaction. In addition, LLLT stimulates a wider dissemination of HGF on the titanium surface. Skinner SM et al. (25) use an impulse Ga-Al-As laser by applying differ- ent energy density levels on fibroblast cultures of human embryos and they have observed a significant rise in collagen levels with irradiated cells. Besides, an enhanced in vitro DNA and ATP synthesis is still demonstrated in the biochemical test area. Laser-assisted increased biostimulation has been reported to cause intracellular metabolic changes, leading as a result to faster cell division, fast matrix production (increased collagen, myofibroblasts, etc.) and cell migration. According to a study conducted by Khadra $\mathrm{M}$ et al. (9), the enhanced initial attachment of the fibroblasts supports the hypothesis that the main therapeutic effect is predominantly associated with the earliest, most sensitive stages of the healing process. Kreisler $\mathrm{M}$ et al. (10) have established a significantly higher proliferative activity 24 after the irradiation, decreasing in time, which shows that a second exposure to laser is necessary for achieving a positive effect regarding cell proliferation. These results concur with the conclusions made by Khadra M, who has confirmed the growth of HGF after exposure to laser treatment for a period of three consecutive days. Other studies verify that LLLT affects the release of specific growth factors by the fibroblasts. Yu W et al. (28) demonstrate that the production of the main fibroblast growth factor (bFGF) by the fibroblasts can be improved by means of laser irradiation. It is obvious that the results of all of these studies are very encouraging and lead the way to generating intact and stable peri-implant soft tissues.

Many researchers believe that LLLT creates conditions which speed up the healing of peri-implant bone defects. Dortbudak O et al. (4) have studied the effect of irradiating osteocytes with low-level laser and the bone resorption with bone implants. The team of scientists demonstrate that osteocyte proliferation was higher with samples that had been exposed to laser irradiation. The speed of bone resorption is not affected by the application of laser. Their results show that there is more vital bone in the irradiated areas than in the areas which were exposed to irradiation, and it can be anticipated that the periimplant bone healing will be accelerated. Guzzardella GA et al. (7) placed ceramic implants in the distal thigh bones of 12 laboratory rabbits. They used a 780 $\mathrm{nm}$ wavelength $\mathrm{Ga}-\mathrm{Al}$-As laser, and the entire energy applied in the test area was $300 \mathrm{~J} \mathrm{~cm}^{2}$. Histomorphometric analysis shows significantly better bone mi- 
cro strength due to the osteocytic proliferation in the group treated with LLLT compared to the reference group. They have also arrived at the conclusion that the postoperative application of LLLT improves the bone-implant interface. Nicolau RA et al. (20) perforated the thigh bones of 48 rats (2 groups), where the group exposed to irradiation was treated with $\mathrm{Ga}$ Al-As laser $\left(660 \mathrm{~nm}, 10 \mathrm{~J} \mathrm{~cm}^{2}\right)$ and radiation exposure on the second, fourth, sixth and eighth day of the postoperative period. They came to the conclusion that LLLT improves bone cell activity (resorption and formation) around the place of restoration without changing bone structure. LLLT particularly enhances osteoclast activity. They presume the inclusion of two mechanisms. First, this laser wavelength stimulates osteoclasts directly. The osteoclasts are multi nucleic sources with many mitochondria with high activity. They found that LLLT used in the period of beginning of the inflammation early after the operation stimulates the increased activity both of osteoclast and of osteoblasts. In addition, Barushka $\mathrm{O}$ et al. (1) and Yaakobi T et al. (27) point out that LLLT is probably a modulator, responsible for the increased levels of alkaline phosphatase in the bone. All these studies found increased amounts of alkaline phosphatase after the application of lowlevel laser. More specifically, they show that irradiation of laboratory animals ensures 50\% higher level of accumulation of calcium compared to the group which was not exposed to irradiation. This finding is of significant importance due to the significance of the adequate and rapid bone growth around the titanium implants. This type of bone formation is one of the most important factors that ensure better stability of implants. As for osseointegration with bone grafts, low-level lasers have been used rarely for enhancing bone formation. Gerbi ME et al. (6) have found that the combined use of lyophilized bovine bone and low-level laser has a positive biostimulating effect on the healing of surgical defects made in the thigh bone of a laboratory rat after 15 and 30 days, and this effect has been attributed above all to LLLT. Pinheiro AL et al. (22) have found that the application of LLLT improves bone formation and increases the quantity of collagen fibres in the area surrounding the graft earlier that the $15^{\text {th }}$ day of the postoperative period. Rochkind S et al. (24) applied low-level laser, individually and in combination with Bio-
Oss, over a period of 14 consecutive days and pointed out that bone healing was improved, and mineralization was better. Ninomiya T et al. (21) have found that LLLT has no effect on bone regeneration during the first 15 days of the postoperative period and that the effect of LLLT depends on time, because the modulating effect is only achieved in the beginning of the differentiation of immature precursors. They have not confirmed the existence of any effect whatsoever during the later stages. Coombe AR et al. (2) confirm this theory by demonstrating that a single dose of LLLT, applied daily over a period of 10 days, was more efficient during the first two postoperative days. A single dose of laser application has no effect on a newly mineralized bone, which means that the effect of LLLT depends on time and dosage and that LLLT causes bone formation through stimulation of immature cells only.

The standpoints reviewed above that LLLT improves bone formation come in contradiction with the opinions of other researchers. Pogrel MA et al. (23) have arrived at the conclusion that no significant improvement is observed in cell proliferation, adhesion or migration, both with fibroblasts and with the keratinocytes, treated with Ga-Al-As laser. According to a recent study conducted by Jakse $\mathrm{N}$ et al. (8), which examines the effect of LLLT on bone healing and osseointegration of titanium implants on a sinus graft model, no confirmation can be made in support of the statement that irradiation with LLLT stimulates bone regeneration. Despite this, scientists have shown that low-level laser therapy could be beneficial for improving implant osseointegration.

Some researchers have suggested that the stimulating effect of LLLT during the first week of the postoperative period could change in an inhibiting effect after this period of time. Barushka $\mathrm{O}$ et al. (1) and Yaakobi T et al. (27) have found that the volumetric fraction of the trabecular bone and the concentration of alkaline phosphatase and calcium in the regenerated experimental bone defects decreases faster in the group which has been exposed to irradiation with He-Ne laser than in the group which has not been exposed to irradiation. The results obtained by Garavello-Freitas I et al. (5), also show that the application of low-level laser for periods longer than 5 minutes does not improve bone reaction regarding the process of bone healing. They suggest that fur- 
ther experiments will have to be carried out in the future in order to establish whether the discontinuation of exposure to irradiation after the first seven days would be more beneficial for bone regeneration.

\section{DISCUSSION}

Dental implantology is the fastest developing specialty in dental medicine. Various methods are used for accomplishing increased osseointegration of implants. The use of LLLT is offered for the purpose of improving soft tissue healing and bone regeneration. Some research shows higher concentration of collagen fibres after exposure if bone defects to low-level laser irradiation. Since collagen is a vital component of the extracellular matrix, the presence of large amounts of collagen fibres would signify increased formation of new bone after matrix mineralization. Besides, it has been established that LLLT improves osteoblast adhesion and vascular migration towards the surface and prepare adequately the implant place for reducing tissue damage. According to information contained in specialized literature, low-energy laser irradiation in doses of 1.5 or $3 \mathrm{~J} \mathrm{~cm}^{2}$ stimulates fibroblasts in vitro. Other studies support the fact that repeated doses, rather than irradiation intensity, are more efficient for the formation of bone and for the implant-tissue interaction. In addition, other authors confirm that the duration of the positive effect of LLLT is not more than one week postoperatively.

\section{CONCLUSION}

It is obvious that there exist some contradictory results in reference to the stimulating effect of LLLT. Some of the inconsistencies can be accounted for by the differences in wavelengths, energy density, exposure time and the surgical procedure. Besides, laser irradiation has a broad impact spectrum on tissues and a single overexposure or lower level exposure may change clinical results significantly. Therefore, adopting the appropriate method of stimulation is of major importance.

\section{REFERENCES}

1. Barushka O, T Yaakobi, U Oron. Effect of low energy laser ( $\mathrm{He}-\mathrm{Ne}$ ) irradiation on the process of bone repair in the rat tibia. Bone. 1995;16:47-55.
2. Coombe AR, CTG Ho, IR Philips. The effects of low level laser irradiation on osteoblastic cells. Clin Orthod Res 2001;4:3-14.

3. Dortbudak O, R Haas, G Mailath-Pokorny. Biostimulation of bone marrow cells with a diode soft laser. Clin Oral Implants Res. 2000;11:540-5.

4. Dortbudak O, R Haas, GM Pokorny. Effect of lowpower laser irradiation on bony implant sites. $\mathrm{Clm}$ Oral Implants Res 2002;13:288-92.

5. Garavello-Freitas I, V Baranauskas, PP Joazeiro, et al. Low-power laser irradiation improves histomorphometrical parameters and bone matrix organization during tibia wound healing in rats. I Photochem Photobiol B 2003;70:81-9.

6. Gerbi ME, ALB Pinheiro, C Marzola, et al. Assessment of bone repair associated with the use of organic bovine bone and membrane irradiated at 830 nm. Photomed Laser Surg 2005;23:382-8.

7. Guzzardella GA, P Torricelli, N Nicoli-Aldini, et al. Osseointegration of endosseous ceramic implants after postoperative low-power laser stimulation: An in vivo comparative study. Clin Oral Implants Res. 2003;14:226-32.

8. Jakse N, M Payer, S Tangl, et al. Influence of lowlevel laser treatment on bone regeneration and osseointegration of dental implants following sinus augmentation: An experimental study on sheep. Clm Oral Implants Res. 2007;18:517-24.

9. Khadra M, N Kasem, SP Lungstadaas, et al. Laser therapy accelerates initial attachment and subsequent behaviour of human oral fibroblasts cultured on titanium implant material. Clin Oral Implants Res. 2005;16:168-75.

10. Kreisler $M, A B$ Christoffers, B Willershausen, et al. Effects of low-level GaAlAs laser irradiation on the proliferation rate of human periodontal ligament fibroblasts in an in vitro study. I Clin Penodontol. 2003;30:353-8.

11. Lalabonova Hr. Studying the opportunities of LLLT for stimulating the healing process of surgical wounds of soft tissues in the oral cavity - methods of application. Physical medicine, rehabilitation, health. 2014;1:19-21.

12. Lalabonova Hr, Hr. Daskalov. Low-level laser therapy in the treatment of aphthae in the oral cavity. Physical medicine, rehabilitation, health. 2010;1:28-9. 
13. Lalabonova Hr. Use of low-intensity laser irradiation in implant dentistry. Journal of IMAB. 2011;17(2):104-6.

14. Lalabonova Hr. Low energy lasers in the management of traumatic ulcers in oral mucosa - methods of application. J of IMAB. 2014 Jan-Jun;20(1):484-6.

15. Lalabonova $H$, Daskalov $H$. Studying the possibilities of the infrared laser with $(\lambda) 904$ for treatment of bone resorption of dental implants in process of osseointegration. J of IMAB. 2014 Jul-Dec;20(3):572-4.

16. Lalabonova $\mathrm{H}$, Daskalov $\mathrm{H}$. Jaw cysts and guided bone regeneration (a late complication after cystectomy of the maxilla). J of IMAB. 2013;19(4):401-3.

17. Lalabonova Hr, Hr Daskalov. Clinical assessment of the therapeutic effect of low level laser therapy on chronic recurrent aphthous stomatitis. J of Biotechnology \& Biotechnological Equipment. 2014. DOI: $10.1080 / 13102818.2014 .966526$.

18. Lalabonova Hr, Ilieva E. Clinical evaluation of the healing process of oral soft tissue surgical wounds stimulated by low-level laser therapy. J of IMAB, 2013;19(2):279-81. DOI:10.5272/jimab.2013192.279.

19. Lalabonova H, Todorov GR, Daskalov H. Clinical assessment of the effect of low level laser treatment of oral mucosa decubitus ulcers. J of IMAB. 2013;19(4):380-382. doi:10.5272/jimab.2013194.380.

20. Nicolau RA, V Jorgetti, I Rigau, et al. Effect of lowpower GaAlAs laser $(660 \mathrm{~nm})$ on bone structure and cell activity: an experimental animal study. Lasers Med Sci. 2003;18:89-94.

21. Ninomiya T, Y Miyamoto, T Ito. High-intensity pulsed laser irradiation accelerates bone formation in metaphyseal trabecular bone in rat femur. J Bone Miner Metab. 2003;21:67-73.

22. Pinheiro AL, FA Limeira Jr, MEM Gerbi. Effect of $830 \mathrm{~nm}$ laser light on the repair of bone defects grafted with inorganic bovine bone and decalcified cortical osseous membrane. I Clin Laser Med Surg. 2003;21:383-8.

23. Pogrel MA, JW Chen, K Zhang. Effects of low-energy gallium-alummum-arsenide laser irradiation on cultured fibroblasts and keratinocytes. Laser Surg Med. 1997;20:426-32.

24. Rochkind S, G Kogan, EG Luger, et al. Molecular structure of the bony tissue after experimental trauma to the mandibular region followed by laser therapy. Photomed Laser Surg. 2004;22:249-53.
25. Skinner SM, JP Gage, PA Wilce. A preliminary study of the effects of laser radiation on collagen metabolism in cell culture. Aust Dent J. 1996;41:188-92.

26. Stein A, D Benayahu, L Maltz, et al. Low level laser irradiation promotes proliferation and differentiation of human osteoblasts in vitro. Photomed Laser Surg. 2005;23:161-6.

27. Yaakobi T, L Maltz, U Oron. Promotion of bone in the cortical bone of the tibia rats by low energy laser (He-Ne) irradiation. Calcif Tissue Int. 1996;59:297-300.

28. Yu W, JO Naim, RJ Lanzafame. The effect or laser irradiation on the release of bFGF from 3T3 fibroblasts. Photochem Photobiol. 1994;59:167-70. 